\title{
Criteria for Endovascular Intervention in Type B Aortic Dissection
}

Sven Zhen Cian Tan ${ }^{1}$, Sidhant Singh ${ }^{1}$, Joaquin Alfonso Palanca ${ }^{1}$, Natasha Austin J ${ }^{1}$, Matti Jubouri $^{2}$, Damian Bailey ${ }^{3}$, Ian Williams ${ }^{4}$, Christoph Nienaber ${ }^{5}$, Joseph Coselli ${ }^{6}$, and Mohamad Bashir ${ }^{7}$

${ }^{1}$ Queen Mary University of London Barts and The London School of Medicine and Dentistry ${ }^{2}$ Hull York Medical School

${ }^{3}$ University of South Wales Faculty of Life Sciences and Education

${ }^{4}$ University Hospital of Wales

${ }^{5}$ Royal Brompton and Harefield Hospitals

${ }^{6}$ Baylor College of Medicine Michael E DeBakey Department of Surgery

${ }^{7}$ NHS Wales Health Education and Improvement Wales

January 7, 2022

\begin{abstract}
Background The use of thoracic endovascular aortic repair (TEVAR) for the management of uncomplicated type B aortic dissection (un-TBAD) remains controversial. There is a lack of consensus over whether pre-emptive TEVAR should be carried out in patients with un-TBAD at risk of progression to complicated TBAD. We present a review of current evidence and seek to suggest criteria where endovascular intervention in un-TBAD may prove beneficial relative to pharmacotherapy alone. Methods and Materials PubMed and Cochrane databases were searched using terms including: type B aortic dissection, risk factors, medical therapy, TEVAR, false lumen expansion, and mortality. Papers were selected based on title and abstract. Results Optimal medical therapy remains the mainstay treatment for patients with un-TBAD, however patients with un-TBAD present with varying degrees of disease progression risk. Factors such as age, aortic morphology, history of connective tissue disorders, false lumen thrombosis, and aortic branch involvement may potentiate progression from un-TBAD to complicated TBAD. Short- and long-term outcomes associated with TEVAR for TBAD remain promising. Conclusion Pre-emptive TEVAR may be beneficial in patients with un-TBAD presenting with the above factors, however further prospective research into the optimal timing for TEVAR in un-TBAD is required.
\end{abstract}

\section{Hosted file}

[Final Copy] Criteria for intervention in TBAD.docx available at https://authorea.com/ users/397612/articles/552034-criteria-for-endovascular-intervention-in-type-b-aorticdissection

\section{Hosted file}

CI TEVAR table risk factors.docx available at https://authorea.com/users/397612/articles/ 552034-criteria-for-endovascular-intervention-in-type-b-aortic-dissection 